UNIVERSIDADE ESTADUAL DE FEIRA DE SANTANA

Autorizada pelo Decreto Federal n ${ }^{0} 77.496$ de 27/04/76

FPpgrydenciamento pelo Decreto $\mathrm{n}^{\circ} 17.228$ de 25/11/2016

PRÓ-REITORIA DE PESQUISA E PÓS-GRADUAÇÃO

COORDENAÇÃO DE INICIAÇÃO CIENTÍFICA

XXIV SEMINÁRIO DE INICIAÇÃO CIENTÍFICA DA UEFS

SEMANA NACIONAL DE CIÊNCIA E TECNOLOGIA - 2020

\title{
UTILIZAÇÃO DE EXTRATO DE RESÍDUO AGROINDUSTRIAL DE ACEROLA COMO ANTIOXIDANTE EM FILMES DE POLI (ÁCIDO LÁTICO)
}

\author{
Hellen Carolina dos Santos Mota ${ }^{\mathbf{1}}$; Geany Peruch Camilloto ${ }^{2}$; \\ 1. Bolsista PIBIC/CNPq, Graduanda em Engenharia de Alimentos, Universidade \\ Estadual de Feira de Santana, e-mail: hellencarolina29@ hotmail.com \\ 2. Orientadora, Departamento de Tecnologia DTEC, Universidade Estadual de Feira de \\ Santana, e-mail: geanyperuch@yahoo.com.br
}

PALAVRAS-CHAVE: Embalagem ativa; Aditivos naturais; Antioxidante.

\section{INTRODUÇÃO}

Ao longo do tempo, à medida que novas necessidades vão surgindo, novas tecnologias e novos produtos passam a ser utilizados no desenvolvimento de embalagens. A crescente preocupação com a segurança alimentar, a extensão da vida de prateleira, a relação custo-eficiência, a conveniência para o consumidor e problemas ambientais, têm impulsionado o desenvolvimento de novas embalagens, bem como de novas matériasprimas para a sua elaboração (REIS, 2011). No contexto da tecnologia de embalagens, surge um novo conceito de embalagens para alimentos, as embalagens ativas, que se baseiam na incorporação de aditivos à embalagem, interagem com a superfície do alimento com o objetivo de melhorar algumas características do produto (SOARES et al., 2009). Estes aditivos podem ter a função de absorver oxigênio, etileno, umidade e odor, emitir dióxido de carbono, agentes antimicrobianos, antioxidantes e aromas (VERMEIREN et al., 1999).

Os antioxidantes frequentemente utilizados em embalagens ativas são hidroxianisol butilado (BHA) e hidroxitolueno butilado (BHT). Entre as vantagens do uso desses antioxidantes sintéticos em embalagens ativas de alimentos estão a alta estabilidade, baixo custo e eficiência, porém, existem preocupações significativas relacionados aos seus aspectos toxicológicos e riscos à saúde. Desta forma, estudos vêm 
sendo realizados visando a utilização de alguns antioxidantes naturais, por exemplo compostos fenólicos, como alternativa aos antioxidantes sintéticos (CHAN et al. 2007; JONGJAREONRAK et al., 2008; YEN et al. 2008).

Os antioxidantes naturais podem ser extraídos de frutas ou de seus resíduos como semente e casca. Há registros de trabalhos na literatura que evidenciam que a acerola é uma excelente fonte de compostos fenólicos (RIBEIRO DA SILVA et al., 2014), estando presente compostos ativos como antocianinas e flavonoides (HANAMURA et al., 2005).

Além da polpa, os resíduos do processamento de acerola também são fontes potenciais de macronutrientes e compostos bioativos, ricos em compostos antioxidantes, como compostos fenólicos, carotenoides e vitamina C. Estudos demonstraram o valor nutricional destes resíduos, com a finalidade de agregar-lhes valor e sugerir novas formas de utilização (SOUSA et al., 2011; ARAÚJO, 2017). O aproveitamento dos resíduos gerados pelo setor agrícola e pela indústria de alimentos é uma das soluções para reduzir possíveis impactos ambientais, bem como agregar valor aos resíduos que são descartados. (PELIZER et al., 2007).

Nesse contexto, o presente trabalho objetivou obter um extrato hidroetanólico do resíduo agroindustrial de acerola e avaliar seu potencial antioxidante a fim de utilizá-lo na produção de embalagens ativas antioxidantes.

\section{MATERIAL E MÉTODOS OU METODOLOGIA (ou equivalente)}

\section{- Preparo das amostras}

O Resíduo Agroindustrial de Acerola (casca e semente) foi gentilmente doado pela Brasfrut, indústria de processamento de polpas de frutas, localizada no distrito de Humildes da cidade de Feira de Santana - BA. O resíduo foi armazenado em freezer a $18^{\circ} \mathrm{C}$ até posterior utilização. O resíduo foi previamente pesado, seco em estufa (MA 033, Marconi) à $45^{\circ} \mathrm{C}$ por aproximadamente $\mathrm{XX}$ dias. Posteriormente, os resíduo seco foi moído e tamizado em peneira de aço inoxidável (ABNT/ASTM 40 - 35 Mesh) para posterior extração.

\section{- Obtenção do extrato}

O extrato de Resíduo Agroindustrial de Acerola foi preparado pelo método de maceração. A partir da amostra de resíduo seco previamente uniformizada, cem gramas (100,0 g) foram pesados em um béquer e adicionadas de um litro (1 L) de solvente hidroetanólico $80 \%$ Etanol/Água, a mistura foi agitada com auxílio de um bastão de vidro 
e seguiu-se a extração por $72 \mathrm{~h}$ em repouso sob ausência de luz. A mistura foi filtrada sob vácuo e o solvente foi evaporado em Rotaevaporador (IKA - RV 10) sob pressão reduzida e temperatura controlada de $60^{\circ} \mathrm{C}$, seguida de evaporação em estufa à $45^{\circ} \mathrm{C}$ até peso constante. A Figura 1 ilustra as etapas do processo de obtenção do extrato.

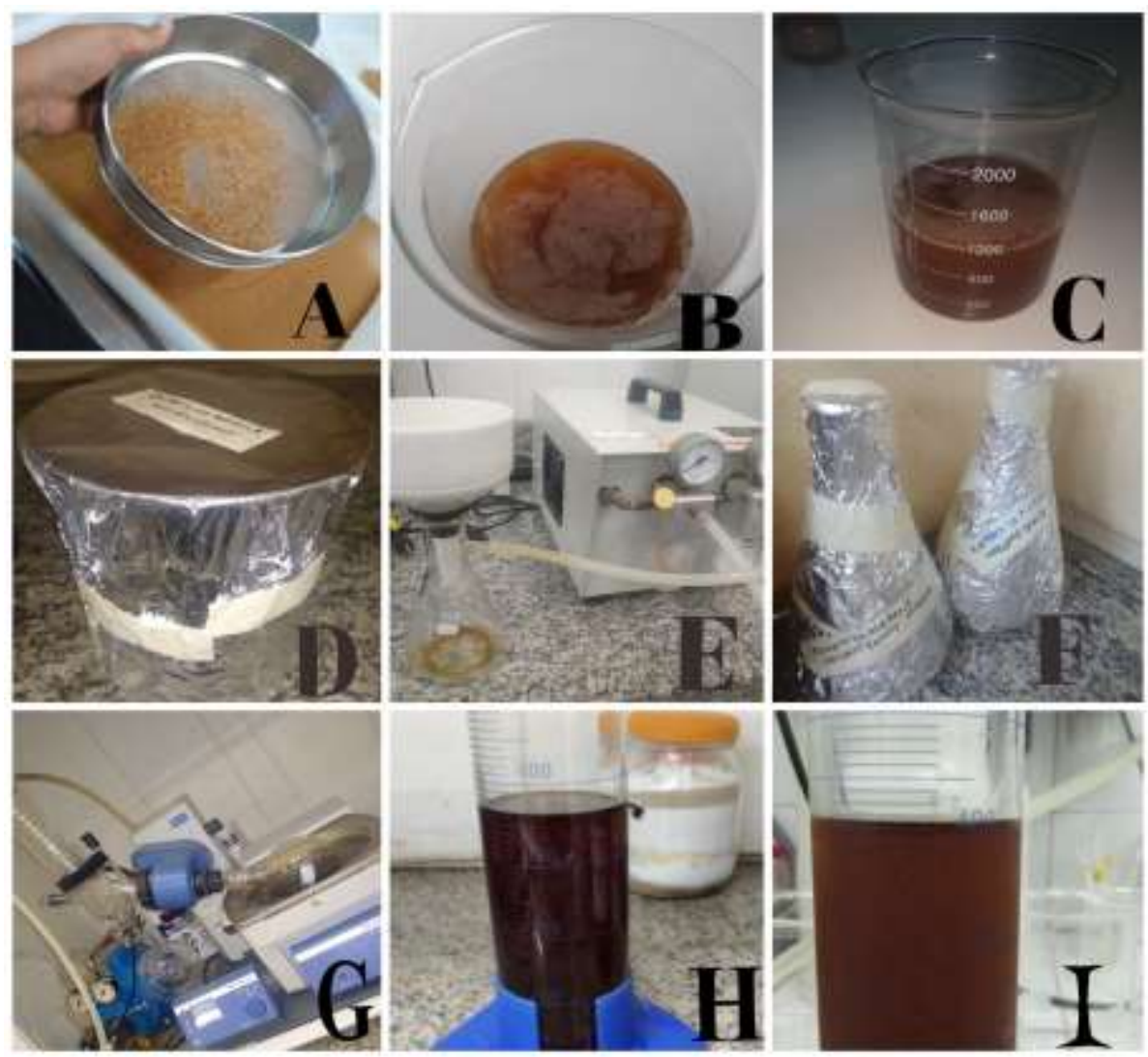

Figura 1: Etapas da elaboração do extrato de acerola. A - Peneiramento do pó triturado; B e C - Elaboração do extrato com álcool etílico PA; D - Utilização do papel alumínio evitando contato do extrato com a luz; E - Filtração a vácuo; F - Armazenamento do extrato filtrado; G - Rotaevaporação; H e I - Extrato rotaevaporado.

\section{- Rendimento}

O rendimento do resíduo seco e do extrato foram determinados de acordo com as Equações 1 e 2.

$$
\begin{aligned}
& \text { Rendimento }_{\text {Resíduo }}=\frac{\text { Resíduo seco }}{\text { Resíduo congelado }} \times 100 \quad(\text { Equação 1) } \\
& \text { Rendimento }_{\text {Extrato }}=\frac{\text { Extrato seco }}{\text { Resíduo } \operatorname{seco}} \times 100 \quad(\text { Equação 2) }
\end{aligned}
$$

- Avaliação do sequestro do radical livre DPPH•

A atividade de sequestro de radical livre do extrato foi avaliado com o uso do radical livre DPPH• (2,2- difenil-1-picril-hidrazil) seguindo a metodologia descrita por Rebaya et al. (2014), com modificações. Para $100 \mu \mathrm{L}$ do extrato, ou padrão, foram 
adicionados $840 \mu \mathrm{L}$ de uma solução de etanol (80\%), seguida da adição de $60 \mu \mathrm{L}$ de uma solução 0,5 mM de $\mathrm{DPPH} \bullet$ (em solução etanólica $80 \%$ ). Após adição do DPPH•, o sistema foi agitado em vórtex e mantido em repouso reagir durante 1 hora, protegido da luz. Diferentes concentrações do extrato $(10-50 \mu \mathrm{g} / \mathrm{mL})$ e da quercetina (padrão) (2 $10 \mu \mathrm{g} / \mathrm{mL}$ ) foram utilizadas nesse ensaio. O branco foi realizado para cada concentração de extrato, ou padrão, com a finalidade de minimizar a interferência dos componentes na leitura das amostras sem adição de DPPH. O controle foi composto de uma mistura de $940 \mu \mathrm{L}$ de uma solução etanólica $(80 \%)$ e $60 \mu \mathrm{L}$ da solução de DPPH• $(0,5 \mathrm{mM})$. Após o tempo de reação, as absorvâncias das amostras foram medidas em um espectrofotômetro UV-VIS Evolution ${ }^{\mathrm{TM}} 220$ (Thermo Scientific) no comprimento de onda de $517 \mathrm{~nm}$.

A porcentagem de inibição das amostras foi calculada a partir dos valores de absorvância pela Equação 3:

$$
\text { \%Inibição }=\left(\frac{A b s_{\text {controle }}-A b s_{\text {teste }}}{A b s_{\text {controle }}}\right) \times 100 \quad \text { Equação } 3
$$

Onde, \%Inibinição é porcentagem de inibição do radical DPPH; $A b s_{\text {controle é a absorvância }}$ do controle e $A b s_{\text {teste }}$ é a absorvância da amostra.

Os gráficos foram gerados plotando a porcentagem de inibição versus a concentração em $\mu \mathrm{g} / \mathrm{mL}$. A equação obtida permitiu calcular o valor do $\mathrm{IC}_{50}$ correspondente à concentração da amostra que reduziu a absorvância inicial do DPPH• em $50 \%$. A as análises foram realizadas em triplicata.

\section{RESULTADOS E/OU DISCUSSÃO (ou Análise e discussão dos resultados)}

A umidade do resíduo agroindustrial de acerola foi de 82,55\%, portanto, o rendimento do resíduo seco foi de $17,45 \%$. O valor de umidade foi semelhante ao repostado na literatura para resíduo de acerola proveniente da agroindústria. Feitosa et al. (2019) e Sousa et al. (2011) encontraram umidade de $83,10 \%$ e $83,45 \%$, respectivamente.

O rendimento do extrato hidroetanólico (\% base seca, $\mathrm{m} / \mathrm{m}$ ) obtidos a partir do resíduo agroindustrial de acerola foi de 7,40\%. O rendimento de extrato pode variar de acordo com a composição química do substrato, pela técnica de extração e pelo tipo de solvente utilizado (DEPKEVICIUS et al., 1998; COSTA et al., 2020). Métodos alternativos de extração vêm sendo estudados objetivo a redução de tempo de processo, melhorias nos rendimentos e na qualidade final do produto (COELHO, 2015). 
Os resíduos de frutas gerados pela agroindústria de frutas geralmente são desperdiçados, no entanto, eles possuem em sua composição vitaminas, minerais, fibras e compostos antioxidantes (LIMA, 2001).

A atividade antioxidante do extrato hidroetanólico do resíduo de acerola foi avaliada pelo método da redução do radical DPPH. O IC 50 do extrato foi de 24,32 $\pm 0,82$ $\mu \mathrm{g} / \mathrm{mL}$. O IC 50 correspondente à concentração de extrato necessária para reduzir o radical $\mathrm{DPPH}$ em $50 \%$, o que significa que valores baixos do $\mathrm{IC}_{50}$ são de antioxidantes potenciais (REBAYA et al., 2014). O IC 50 da quercetina, padrão utilizado, foi de $8,81 \pm 0,15 \mu \mathrm{g} / \mathrm{mL}$. Por se tratar de um flavonoide puro, o potencial antioxidante da quercetina é maior que do extrato. Sousa et al. (2011) relataram valor de $\mathrm{IC}_{50}$ de $308,07 \pm 0,75 \mu \mathrm{g} / \mathrm{mL}$ para extrato hidroalcóolico do resíduo de acerola. O menor poder antioxidante do extrato obtido por Sousa et al. (2011) quando comparado ao deste estudo, pode ser justificada pela ausência da etapa secagem/concentração do extrato.

Cefali et al. (2018) estudaram extrato hidroetanólico de polpa de acerola e obtiveram $90 \%$ de inibição de radicais livres na concentração de $0,01 \mathrm{~mL} / \mathrm{mL}$ no teste de atividade antioxidante in vitro.

\section{CONSIDERAÇÕES FINAIS (ou Conclusão)}

O extrato hidroetanólico obtido do resíduo agroindustrial de acerola exibiram ação antioxidante, demonstrando que este resíduo pode ser utilizado como fonte de antioxidantes naturais e que o processo de extração de compostos bioativos foi eficiente. A utilização de resíduos para obtenção de compostos bioativos contribui para a diminuição da contaminação ambiental que o excesso desses resíduos vem causando.

Diante disso, o extrato apresenta potencial para utilização em materiais poliméricos objetivando a produção de embalagens ativas antioxidantes.

\section{REFERÊNCIAS}

ARAÚJO, I. M. C. Caracterização bioativa de resíduos de frutas tropicais. 2017. $45 \mathrm{f}$. Trabalho de Conclusão de Curso (Graduação em Nutrição) - Curso de Nutrição, Universidade Federal do Rio Grande do Norte, Natal, 2017.

CEFALI, L.C. et al. Vitamin C in Acerola and Red Plum Extracts: Quantification via HPLC, in Vitro Antioxidant Activity, and Stability of their Gel and Emulsion Formulations. Journal of AOAC International, v. 101, n. 5, p. 1461-1465, 2018.

CHAN, E. W. C. et al. Antioxidant activity of Camellia sinensis leaves and tea from a lowland plantation in Malaysia. Food Chemistry, v. 102, p. 1214-1222, 2007. 
COELHO, R. A. Obtenção de óleo de sementes de quiuí (actinidia deliciosa) utilizando extração com solvente pressurizado e extração assistida com ultrassom. 75f. Tese (Doutorado em Engenharia de Alimentos), Universidade Federal do Paraná, Curitiba, PR, 2015.

COSTA, B.S.L. et al. Otimização da extração assistida por ultrassom de compostos fenólicos totais e flavonoides a partir dos frutos de acerola (Malpighia sp.). Revista Brasileira Militar de Ciências, v. 6, n. 14, p. 39-46, 2020.

DEPKEVICIUS, A., et al. Antioxidant activity of extracts obtained by different isolation procedures from some aromatic herbs grown in Lithuania. Journal of the Science of Food and Agriculture, v. 77, p. 140-146, 1998.

FEITOSA, B.F. et al. Cinética de secagem dos resíduos da agroindústria processadora de polpa de frutas. Energia na Agricultura, v. 34, n. 1, p. 134-141, 2019.

HANAMURA, T. et al. Structural and functional characterization of polyphenols isolated from acerola (Malpighia emarginata DC.) fruit. Bioscience, Biotechnology, and Biochemistry, v. 69, p. 280-286, 2005.

JONGJAREONRAK, A. et al. Antioxidant activity and properties of fish skin gelatin films incorporated with BHT and atocopherol. Food Hydrocolloids, v. 22, p. 449-458, 2008.

LIMA, L. M. O. Estudo do Aproveitamento dos Bagaços de Frutas Tropicais, Visando a Extração de Fibras. 2001. 108 f. Dissertação (Mestrado em Engenharia Química)-Universidade Federal do Rio Grande do Norte. Natal.

PELIZER, L. H. et al. O. Utilização de resíduos agroindustriais em Processos Biotecnológicos como perspectiva de redução do Impacto Ambiental. Journal Technology Management Innovation, v. 2, p.118-127, 2007.

REBAYA, A. et al. Total Phenolic, Total Flavonoid, Tannin Content, and Antioxidant Capacity of Halimium halimifolium (Cistaceae). Journal of Applied Pharmaceutical Science, v. 5, n .1, p. 52-57. 2015.

REIS, L, C, B. Formulação e caracterização de filmes biodegradáveis de fécula de mandioca incorporados com polpa de manga e extrato de erva-mate, e seu efeito na preservação de alimentos. Dissertação (mestrado) - Universidade Federal da Bahia, Faculdade de Farmácia, Salvador, 2011.

RIBEIRO DA SILVA, L. M et al. Quantification of bioactive compounds in pulps and by-products of tropical fruits from Brazil. Food Chemistry, v.143, p. 398-404, 2014.

SOARES, N. F. F. et al. Novos desenvolvimentos e aplicações em embalagens de alimentos. Ceres, v. 56, p. 370-378, 2009.

SOUSA, M. S. B. et al. Caracterização nutricional e compostos antioxidantes em resíduos de polpas de frutas tropicais. Ciência e Agrotecnologia, v. 35, p. 554-559, 2011.

SOUSA, M.S.B. et al. Caracterização nutricional e compostos antioxidantes em resíduos de polpas de frutas tropicais. Ciência e agrotecnologia, v. 35, n. 3, p. 554-559, 2011.

VERMEIREN, L. et al. Development in the active packaging of food. Trends in Food Science \& Tecnology, v. 10, p. 77-86, 1999.

YEN, M. et al. Antioxidant properties of chitosan from crab shells. Carbohydrate Polymers, v. 74, p. 840-844, 2008. 